\title{
Enredados con la sierra. Las plantas en las estrategias sostenibles de sobrevivencia del grupo indígena Guarijío/Makurawe de Sonora, México
}

Entangled in the sierra. Plants uses as a sustainable strategy of survival among the Guarijio/Makurawe people of Sonora, Mexico

Noemí Bañuelos-Flores ${ }^{1}$, Patricia L. Salido-Araiza²

Banuelos-Flores, N; Salido-Araiza, P. L. Enredados con la sierra. Las plantas en las estrategias sostenibles de sobrevivencia del grupo indígena Guarijío/Makurawe de Sonora, México. Tecnología en Marcha. Vol. 33-1. Enero-Marzo 2020.

Pág 178-192.

doi) https://doi.org/10.18845/tm.v33i1.3849

1 Profesora Investigadora, Centro de Investigación en Alimentación y Desarrollo A.C

(CIAD). Coordinación de Desarrollo Regional. Hermosillo, Sonora. México. Correo electrónico: noemi@ciad.mx

2 Profesora Investigadora, Centro de Investigación en Alimentación y Desarrollo A.C

(CIAD). Coordinación de Desarrollo Regional. Hermosillo, Sonora. México. Correo electrónico: psalido@ciad.mx 
Palabras clave

Etnobotánica; Grupos indígenas; Conocimiento tradicional; Guarijío/Makurawe; Sonora.

\section{Resumen}

Los Guarijío/Makurawe de Sonora representan uno de los pueblos indígenas más olvidados en el noroeste de México. El presente trabajo analiza desde una perspectiva etnobotánica la importancia que para este grupo indígena guardan los recursos vegetales, a partir del examen de sus distintas formas de aprovechamiento. La metodología utilizada se apoyó en la aplicación de entrevistas personales a profundidad, observación directa y participativa así como talleres comunitarios. Las personas de mayor edad, amas de casa, trabajadores del campo, artesanos, curanderos, rezandero, promotor cultural bilingüe y el gobernador del pueblo, fueron actores claves en la búsqueda de información. Algunos resultados revelan que los Guarijío personifican un ejemplo de sobrevivencia con base en las formas de uso de su patrimonio biocultural. Los saberes indígenas respecto a los recursos vegetales, representan las raíces desde donde debe plantearse cualquier propuesta de desarrollo sostenible que busque mejorar las condiciones de bienestar de este pueblo.

\section{Keywords}

Ethnobotany; indigenous groups; traditional knowledge; Guarijio/Makurawe; Sonora.

\section{Abstract}

The Guarijio/Makurawe people of Sonora are one of the most ignored native groups in Mexico's Northwest region. From an ethnobotanical perspective, this paper analyzes the importance of plant resources for this indigenous community, according to their different uses. Some methodological technics included in depth personal interviews, direct-participative observation as well as community workshops. Among key actors interviewed included the eldest people, housewives, rural workers, artisans, healers, cultural promotor, and the local governor. Results show that the Guarijio exemplify a case of subsistence based on the uses of their biocultural heritage. Indigenous knowledges on vegetal resources represent the roots for any proposal of sustainable development focused on the improvement of wellbeing of this people.

\section{Introducción}

Las plantas son seres vivos generosos, esenciales para la sostenibilidad de la Tierra; se enredan con facilidad en diversos espacios de la vida cotidiana de los grupos humanos: alimentan, curan, protegen e invaden sus viviendas, su trabajo, sus ceremonias y se sitúan en el centro mismo de su existencia. Los servicios materiales e inmateriales que proporcionan son innumerables e inconmensurables, de modo tal que cuesta trabajo pensar en alguna necesidad humana donde la llamada cubierta verde no esté presente. Algunos autores [1] ponen de manifiesto que México, junto con Indonesia, India, Australia, Zaire y Brasil son seis de los países más privilegiados porque en sus territorios se enlazan alta diversidad biológica y cultural. A pesar de ello, México presenta uno de los mayores índices de deforestación a nivel mundial: "en lo que va del siglo XXI, ha perdido alrededor de 2.5 millones de hectáreas de bosques y ha recuperado solamente 633,000; lo que genera un balance negativo de 1 millón 840,000 hectáreas perdidas entre el 2000 y 2012"[2]. 
Hoy día, cuando observamos que los recursos naturales, como parte substancial de las bases materiales del desarrollo, se han degradado y que la pobreza aumenta alarmantemente, junto a procesos demográficos preocupantes, resulta apremiante identificar y rescatar aquellos conocimientos que contribuyan a resaltar la utilización sustentable de estos recursos. En gran parte del territorio mexicano existen diversos grupos indígenas que han sabido convivir con la naturaleza de una manera diferente, es decir, con una concepción integral y armónica con su entorno. Entre las comunidades indígenas existe una lógica de utilización de los recursos naturales cuyo reconocimiento resulta estratégico, especialmente para paises como el nuestro, con un patrimonio biocultural tan rico. Sin lugar a dudas, a nivel nacional existen abundantes investigaciones etnobotánicas especialmente enfocadas a las regiones centro y sur. Sin embargo, este tipo de estudios en Sonora son escasos; con algunas valiosas excepciones, los recursos vegetales aparecen como escindidos de los grupos humanos y desde un enfoque unidimensional. Una razón más que justifica el presente trabajo es que desde finales del año 2010 a la fecha, el pueblo Makurawe/Guarijío de Sonora está siendo amenazado con la pérdida de sus recursos y sus formas de vida, su lengua, su conocimiento tradicional, a causa de la eventual puesta en marcha del proyecto de construcción de la presa Ilamada Los Pilares/ Bicentenario, en las inmediaciones del poblado de San Bernardo, municipio de Álamos. En este sentido, hoy más que nunca resulta imperativo y especialmente estratégico para México reconocer el patrimonio biocultural de los pueblos indígenas [1] [3].

\section{Metodología}

La investigación se llevó a cabo en Mesa Colorada, por su ubicación central y por ser la localidad Guarijío más importante en términos de población. Se trata de un espacio social de reunión y discusión de los principales problemas que afectan a este pueblo indígena. La comunidad está inserta en una región intercultural --donde interactúan mestizos e indígenas-en constante contacto con instituciones oficiales. Las personas de mayor edad, amas de casa, trabajadores del campo, artesanos, curanderos, rezandero, promotor cultural bilingüe y el gobernador del pueblo, fueron actores claves en la búsqueda de información. Para ello se realizaron entrevistas personales a profundidad, observación directa y participativa así como talleres comunitarios. El propósito de estos últimos fue el escuchar directamente la voz de la comunidad acerca del significado y la importancia que revisten los recursos vegetales en sus formas de vida como grupo; así como sirvieran como un espacio de reflexión sobre los problemas socioeconómicos y medioambientales que enfrentan hoy día y las propuestas alternativas a partir de los recursos disponibles. La metodología utilizada para este tipo de talleres se caracteriza por llevarse a cabo con un enfoque participativo y endógeno. La identificación, valoración y la jerarquización del objeto de análisis (recursos vegetales) es hecha directamente por los participantes, buscando alcanzar consensos en las decisiones. Esta acción intenta potenciar los recursos local-comunitarios (humanos, materiales y naturales) para fomentar el desarrollo regional sustentable. Los talleres comunitarios se llevaron a cabo mediante la actividad de lluvia de ideas y ejercicio de priorización.

Se recurrió a las nociones de etnobotánica/saberes indígenas, identidad/cultura y territorio/ región, como concepciones nodales integradoras que permitieron tejer hilos conductores entre los aspectos biológicos, sociales, económicos y culturales (figura 1). Las investigaciones sobre recursos naturales que se realizan en zonas marginadas, requieren de la participación de los pobladores, actores sociales -indígenas o campesinos- quienes deben ser considerados no sólo como fuentes de información sino como agentes centrales con capacidades para opinar, proponer y decidir sobre sus propios problemas. 


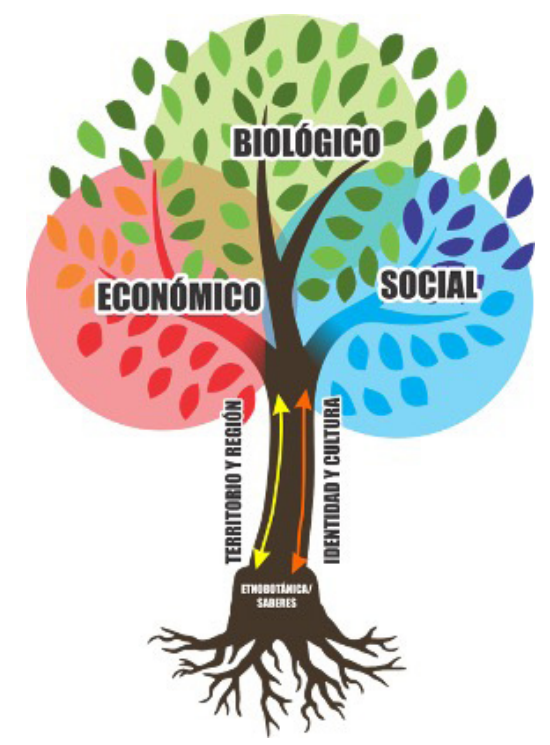

Figura 1. Hacia una propuesta productiva. Con los pies en el territorio.

Los que le danzan a la tierra. Un rostro indígena apenas visible

Existen dos grupos indígenas Guarijío, asentados en una zona de la Sierra Madre Occidental, en los límites de Sonora y de Chihuahua, estados a los que se atribuye la separación de la vida política, administrativa y social del territorio histórico Guarijío [4]. El Censo de Población del Instituto Nacional de Estadística Geografía e Informática [5] estimó un total de 2,136 hablantes de lengua Guarijío mayores de 5 años en el país, distribuidos en los estados mencionados [6]. Esta lengua se ha definido como perteneciente al grupo nahua-cuitlateco, del tronco yutonahua, familia pimacora, subdivisión cahita-tarahumara, en su expresión lingüística Guarijío [4]

Los Guarijío ubicados en Sonora, forman parte de los ocho grupos indígenas considerados como nativos de esta entidad, junto a los Mayo, Yaqui, Pima, Seri, Pápago, Cucapá y Kikapu. Los Guarijío de Sonora se denominan a sí mismos como Makurawe, que significa "Los que agarraron las piedras o los que se agarraron de las manos y bailan"[7], y están emparentados, histórica y culturalmente con los Raramuri y los Yoreme Mayo. Residen principalmente en los municipios de Álamos y El Quiriego, hacia el sur del estado (figura 2). La población está distribuida de manera muy dispersa en 22 localidades que integran 3 ejidos. Destacan los poblados de Mesa Colorada, Los Bajíos y Guajaray, como los de mayor población.

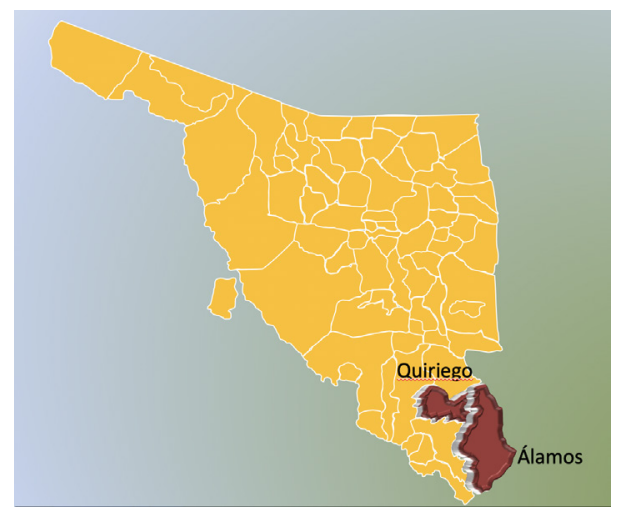

Figura 2. Localización geográfica de la región Makurawe/guarijío de Sonora. 
Los Guarijío son representados por sus gobernadores, como autoridad máxima, estructura propuesta e impulsada por grupos y organismos indigenistas en sus actividades por la región, probablemente desde finales de los años 1970s. Los Guarijío, "Son en parte producto de una serie de despojos, olvidos, y abandonos que los han mantenido al margen de la historia y el desarrollo étnico por un largo tiempo. Su historia, sus tradiciones, creencias, forma de vida han sido ignoradas, lo que les ha obligado a sobrevivir y resistir." [8].

Las condiciones de pobreza y marginación que prevalecen en el territorio guarijío son aún más difíciles que las enfrentadas en el resto de los municipios donde se ubican. Aunado a ello, la accidentada geografía de su territorio, la dispersión de la población, la presencia de suelos pobres, escasa precipitación pluvial, etc., en conjunto, limitan no solamente las actividades que tradicionalmente ahí se desempeñan, sino también sus posibilidades de reproducción biológica y cultural. Los índices regionales de marginación se multiplican, ante la insuficiente red de servicios regulares de agua potable, electrificación, vías adecuadas de comunicación carretera y telefónica, etc., aspectos vinculados conjuntamente con su problemática de educación, alimentación y salud.

En contraste, los Guarijío tienen la fortuna de encontrarse asentados en una área rica en diversidad biológica. Según algunos expertos, su territorio es una zona de transición donde se conjugan especies de los ecosistemas desérticos y tropicales. Ahí se encuentra la selva baja caducifolia mejor conservada del continenete americano. [9] Por su importancia en términos de la distribución geográfica del bosque tropical caducifolio en México, la Sierra de ÁlamosRío Cuchujaqui fue decretada en 1996 como Área de Protección de Flora y Fauna Silvestre y Acuática, a fin de salvaguardar una extensa área de bosque tropical caducifolio en Sonora [10]. En 2007, esta reserva fue incorporada a la Red Mundial de Reservas de Biosfera de la UNESCO.

Debido a que el 90 por ciento de su territorio (de un total de 24,318 has) se clasifica como de agostadero cerril, la ganadería se ha constituido desde hace ya algunas décadas en la principal actividad económica [11]. La agricultura es de temporal, para autoconsumo, destacando el maíz, fríjol y calabaza como principales cultivos. También, en las vegas del Río Mayo, o de los arroyos, algunas familias siembran maíz, sandía, cebolla, melón, tomate, frijol, calabaza, rábano, entre otros. El monte y el río siguen representando una fuente importante de alimentos a través de la caza, la pesca y la recolección. En suma, los guarijío han construido su vida en estrecha simbiosis con su entorno natural, enredados con la sierra, constituyendo un ejemplo de sobrevivencia biológica y cultural. Aquí en este espacio físico, han edificado su cultura, su economía, su identidad, su sentido de pertenencia y sus saberes.

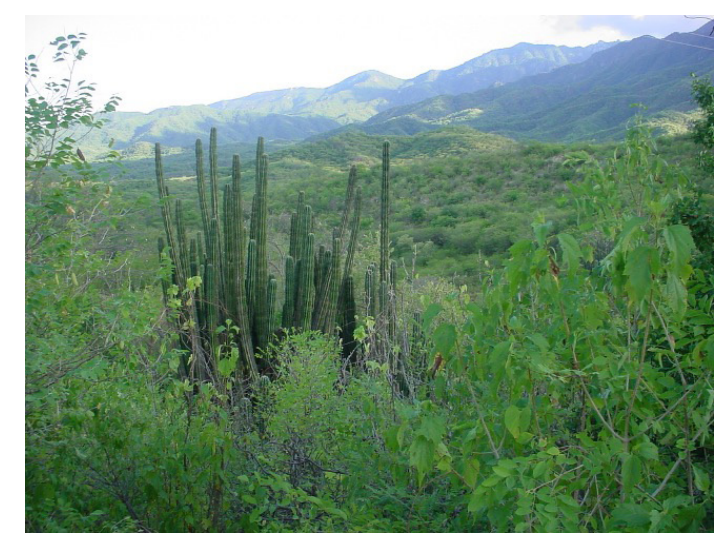

Figura 3. Selva baja caducifolia, Álamos, Sonora. 


\section{Resultados}

La sierra y los saberes Makurawe/Guarijío. Existen pueblos que desde hace muchos años han aprendido a vivir con la naturaleza y con la sociedad de una manera diferente, quizá más sabia, en términos ecológicos. Sin embargo, aún cuesta trabajo reconocerlo. Es difícil encontrar tres etnias tan relacionadas con sus entornos como los Tarahumaras con el bosque de pinos, los Mayo con la vegetación espinosa y los Guarijío con los arbustos [12]. Esta interacción tan estrecha con su medio natural parte de la idea de la ubicación del hombre en la naturaleza, pensada como una relación armónica: la naturaleza no es su enemigo, por lo tanto no hay que vencerla; más bien hay que conocerla y aprender de ella. Los Guarijío/Makurawe han aprendido a vivir con la sierra y no en ella; se consideran una especie más de su territorio y no precisamente la más importante, por eso le cantan a sus hermanos: el mapache, el maguey, la iguana, el tecolote, el coyote, el pájaro carpintero y el palo verde; le danzan a la tierra, y a la lluvia. Para este pueblo, conocer la sierra y las especies que en ella habitan, así como sus ciclos reproductivos, ha representado una sabia estrategia de supervivencia biológica y cultural. El monte, el río, la lluvia, y todas las especies que en la sierra viven tienen un significado profundo dentro de su memoria colectiva. La sierra no es sólo un espacio geográfico contenedor de materias primas; "El entorno comunitario aparece en la memoria indígena como un ámbito cargado de significaciones que la persona va reconociendo y registrando a través de la vida como un espacio de amplio sentido y datos de su percepción, en el monte, los cerros, las cuevas, el río: lugares sagrados y vitales con los cuales la humanidad indígena interactúa siempre con respeto" [13].

Sin duda alguna, vivir con la sierra no ha sido fácil; ha significado un largo proceso que implica gran resiliencia, sabiduría y creatividad. Los Guarijío han demostrado ser uno de los grupos indígenas sonorenses más tenaces que han aprendido a vivir y desarrollar una gran capacidad de adaptación creativa en un territorio diverso, complejo y cambiante. Ahí, en la sierra, han construido su cultura, su economía, su identidad, su sentido de pertenencia y sus saberes.

Enredados con las plantas. Las plantas, como parte esencial de la sierra, siguen siendo en la actualidad elementos esenciales en su permanencia biológica y cultural: alimentan, curan, protegen, invaden su vivienda, su trabajo, sus fiestas y ceremonias religiosas. Observar las distintas formas de utilización de los recursos vegetales es como descorrer una cortina hacia la percepción del mundo Guarijío. Significa conocer y entender la representación que la sociedad Guarijío tiene de ella misma, de su historia y devenir en relación con el mundo que habitan. En la sierra, han sabido encontrar una variada "farmacia", un mercado al aire libre lleno de frutos, enseres y herramientas de trabajo. La sierra es una escuela a cielo abierto, sin paredes, un laboratorio en donde prueban y descubren las cualidades de los recursos vivos y los ciclos reproductivos.

Los Guarijío, son obstinados observadores de su entorno natural. Distinguen con facilidad una gran diversidad de especies de los distintos tipos de microclimas y saben qué parte usar y en qué temporada aprovecharlas. Dentro de sus ceremonias religiosas, las plantas son elementos imprescindibles. La guásima (Guazuma ulmifolia) no sólo es útil para construir sus viviendas o quitar el hambre. En las ceremonias esta planta ha sido transformada en cruz, símbolo religioso central en sus fiestas. El etcho, (Pachycereus pecten-aboriginum) el torote (Bursera laxiflora) y el bule (Lagenaria sicerar) convertidos en arpa, máscara de pascola y sonaja, cantan y bailan en las ceremonias; el aromático copal (Bursera lancifolia) purifica este espacio y los cigarros de macucho (Nicotiana rustica) elaborados con hoja de maíz, son una forma de comunicación con los espíritus.

En actividades como la pesca y la caza, las manos guarijías tejen ingeniosas cimbras y trampas naturales con las fibras y ramas de algunas plantas como el batamote (Bacharis salicifolia) y 
algunos agaves; también entrelazan las hojas de la palma (Sabal uresana) para convertirlas en frescos petates, guaris y sombreros. Sus cantos, cuentos y leyendas están inspirados en los seres vivos que forman parte de este complejo sistema natural que es la sierra.

Alimentarse y hacer frente a la enfermedad son las necesidades más básicas de todo ser humano, en este sentido la sierra representa el espacio esencial para cubrirlas.

De frutos, hojas y raíces. Los Guarijío conocen una gran diversidad de especies que aún forman parte esencial de su dieta, incluyendo frutos, raíces y semillas. Algunas de las especies más importantes se muestran en el siguiente cuadro.

Cuadro 1. Las estructuras vegetales útiles en la alimentación de los makurawe/guarijio de Sonora.

\begin{tabular}{|c|c|c|c|c|c|c|c|c|c|}
\hline Nombre común & $\begin{array}{l}\text { Nombre } \\
\text { makurawe }\end{array}$ & Nombre científico & Fruto & Flor & Hoja & Tallo & Raíz & Semilla & Corteza \\
\hline Arrellán & Choqué'i & $\begin{array}{l}\text { Psidium sartorianum (O. } \\
\text { Berg) Nied. }\end{array}$ & $x$ & & & & & & \\
\hline Chalate & Guagüurí & Ficus trigonata L. & $x$ & & & & & & \\
\hline Chani & Comba'ari & Hyptis suaveolens (L.)Poit. & $x$ & & & & & & \\
\hline Chichiquelite & Maneyoqui & Solanum americanum Miller & $x$ & & $x$ & & $x$ & & \\
\hline Chichivo & Chichihuó & Dioscorea remotiflora Kunth & & & & & $x$ & & \\
\hline Chiltepín & Co’co’rí & $\begin{array}{l}\text { Capsicum annum var. } \\
\text { glabriusculum (Heiser y } \\
\text { Pickersgill) }\end{array}$ & $x$ & & & & & & \\
\hline Encino & Cusi & Quercus albocinata Trel. & $x$ & & & & & & \\
\hline Etcho & Chiquí & $\begin{array}{l}\text { Pachuycereus pecten- } \\
\text { aboriginum Engelm. ex S. } \\
\text { WatsonBritton \& Rose }\end{array}$ & $x$ & & & & & $x$ & \\
\hline Guamúchil & Maicochini & $\begin{array}{l}\text { Pithecellobium dulce (Roxb.) } \\
\text { Benth. }\end{array}$ & $x$ & & & & & & \\
\hline Guásima & Agiyá & Guazuma ulmifolia Lam. & $x$ & & & & & $x$ & \\
\hline Huehue & Huehue & $\begin{array}{c}\text { Amaranthus leucocarpus } \\
\text { S.Watson }\end{array}$ & & & & & & $x$ & \\
\hline Igualama & Juhuari & Vitex mollis Kunth & $x$ & & & & & & \\
\hline Lechuguilla & Magí & Agave angustifolia Haw. & & $x$ & $x$ & $x$ & & & \\
\hline Mauto & Ma'a & $\begin{array}{l}\text { Lysiloma divaricatum (Jacq.) } \\
\text { J.F.Macbr. }\end{array}$ & $x$ & & & & & & \\
\hline Mezquite & Jupa'ara & Prosopis glandulosa Torr. & $x$ & & & & & & \\
\hline Mostaza & Guacharay & $\begin{array}{c}\text { Dryopetalon runcinatum A. } \\
\text { Gray }\end{array}$ & & & $x$ & & & $x$ & \\
\hline Nopal & Nopá & $\begin{array}{c}\text { Opuntia cf. Wilcoxii Brit \& } \\
\text { Rose }\end{array}$ & $x$ & & $x$ & & & & \\
\hline Palo mulato & Mulato & $\begin{array}{c}\text { Bursera grandifolia (Schltdl.) } \\
\text { Engl. }\end{array}$ & & & & & & & $x$ \\
\hline $\begin{array}{l}\text { Papache } \\
\text { borracho }\end{array}$ & Cabijósocura & Randia obcordata S. Watson & $x$ & & & & & & \\
\hline Papache & Josocura & $\begin{array}{l}\text { Randia echinocarpa Mocino } \\
\text { \& Sesse ex DC. }\end{array}$ & $x$ & & & & & & \\
\hline Pochote & Guacapí & $\begin{array}{c}\text { Ceiba acuminata (S. Watson) } \\
\text { Rose }\end{array}$ & & & & & $x$ & $x$ & $x$ \\
\hline
\end{tabular}

Continúa... 
Continación

\begin{tabular}{|c|c|c|c|c|c|c|c|c|c|}
\hline Nombre común & $\begin{array}{l}\text { Nombre } \\
\text { makurawe }\end{array}$ & Nombre científico & Fruto & Flor & Hoja & Tallo & Raíz & Semilla & Corteza \\
\hline Quelite & Huehué & Amaranthus palmeri S. Wats. & & & $x$ & & & & \\
\hline Sahui & Saguí & Panicum hirticaule J. Presl & & & & & & $x$ & \\
\hline Saya & Sayú & $\begin{array}{l}\text { Amoreuxia cf. palmatifida } \\
\text { Sesse \& Mocino ex DC. }\end{array}$ & $x$ & $x$ & & & $x$ & $x$ & \\
\hline Sibiri & Sevelí, sahueri & Opuntia thurberi Engelm. & $x$ & & $x$ & & & & \\
\hline Sotol & Selé & $\begin{array}{c}\text { Dasylirion wheeleri S. Watson } \\
\text { ex Rothr. }\end{array}$ & & & & $x$ & & & \\
\hline Tchuna & Huilocochi & Ficus insipida Willd. & $x$ & & & & & & \\
\hline Tempisque & Cajé & $\begin{array}{c}\text { Sideroxylon tepicense (Pittier) } \\
\text { T.D. }\end{array}$ & $x$ & & & & & & \\
\hline Tepeguaje & Machahuí & Lysiloma watsonii Rose & & & & & & $x$ & \\
\hline Totales & & & 19 & 2 & 6 & 2 & 4 & 8 & 2 \\
\hline
\end{tabular}

Aunque en la actualidad las plantas siguen siendo importantes, es interesante señalar que la alimentación es un aspecto que evidentemente está sufriendo modificaciones lentas, pero constantes, especialmente en la población de jóvenes y niños. Durante la realización de trabajo de campo en la región fue posible observar la llegada de camiones cargados de una variedad de alimentos que incluían verduras, frutas, harinas, carne de res, pescado, pollo y cerveza. Sin embargo, llamaba la atención que junto a estos productos resaltaba una gran cantidad de paquetes de frituras y refrescos de cola, que ocupaban más de la mitad del espacio de carga del vehículo.

El problema no es que hoy día los Guarijío puedan acceder a otro tipo de alimentos, sino que muchos de éstos no tienen las cualidades alimenticias naturales que ofrecería un fruto de papachi o una fresca raíz de chichivo, y que finalmente, su substitución pudiera estar afectando negativamente la salud, especialmente la de la población joven. Cuesta trabajo pensar que siendo el grupo indígena Guarijío el que tiene los índices más altos de marginación y pobreza entre los demás grupos étnicos de Sonora, se observen casos excepcionales de niños con problemas de obesidad. Con base en nuestros primeros acercamientos a este grupo indígena, en la década de los 1980s, pudiéramos pensar que esta situación no se observaba antes con tanta intensidad.

La medicina tradicional, otro encuentro con la sierra. Los guarijío han sabido encontrar en la sierra elementos esenciales para hacer frente a la enfermedad y la muerte: plantas, animales y minerales son los recursos más utilizados. Al igual que otros grupos, han desarrollado un cuerpo teórico práctico de conocimientos alrededor de las plantas y de las formas de utilización; han aprendido a diferenciar las especies, saben cuándo y cómo cortarlas, además distinguen qué estructura vegetal es la más adecuada para utilizar en cada tipo de padecimiento.

La medicina tradicional se lleva a cabo en dos espacios esenciales: el familiar, donde la madre es la encargada de velar por la salud de sus familiares, y el comunal, donde concurren personajes conocidos como curanderos o ioowiames, quienes son especialistas en tratar distintas enfermedades: parteros, sobadores, hueseros, y que se encargan también de aliviar enfermedades espirituales como el "mal puesto". 
Cuadro 2. Las plantas en la medicina tradicional makurawe/guarijío de Sonora.

\begin{tabular}{|c|c|c|c|c|}
\hline Nombre común & $\begin{array}{l}\text { Nombre } \\
\text { makurawe }\end{array}$ & Nombre científico & Enfermedad & Parte útil \\
\hline Algodoncio & Machicari & Wimmeria mexicana (DC.) Lundell & Resfriado & Corteza \\
\hline Amapa & Tabuió & $\begin{array}{l}\text { Tabebuia impetiginosa (Mart. ex DC.) } \\
\text { Standl. }\end{array}$ & Má de orin & Tallo \\
\hline Baiburia & Nacachíchicore & Dorstenia drakeana $\mathrm{L}$. & $\begin{array}{c}\text { Fiebre, susto, dolor de muela y } \\
\text { oído }\end{array}$ & Raíz \\
\hline Batamote & Guachomó & $\begin{array}{c}\text { Baccharis salicifolia (Ruiz \& Pavon) } \\
\text { Persoon }\end{array}$ & $\begin{array}{l}\text { Mal de orín, dolor de cabeza y } \\
\text { fiebre }\end{array}$ & Raíz, hojas \\
\hline Biznaguita & Hue tchurí & $\begin{array}{c}\text { Echinocereus stoloniferus W.T. } \\
\text { Marshall }\end{array}$ & Dolor de oído & Tallo \\
\hline Cacachila & Jimuari, imora & $\begin{array}{l}\text { Karwinskia humboldtiana (Willd. ex } \\
\text { Roemer \& Schultes) Zucc. }\end{array}$ & $\begin{array}{l}\text { Dolor de higado, mal de orín y } \\
\text { gastritis }\end{array}$ & Tallo \\
\hline Chani & Comba'ari & Hyptis suaveolens (L.)Poit. & Extreñimiento & Raíz \\
\hline Chicura & Chicurá & $\begin{array}{c}\text { Ambrosia ambrosioides (Cav.) } \\
\text { W.W.Payne }\end{array}$ & Desechos de la matríz, parto & Raíz \\
\hline Chiltepín & Co'co'rí & $\begin{array}{l}\text { Capsicum annum var. glabriusculum } \\
\text { (Heiser y Pickersgill) }\end{array}$ & "Mal puesto" & Frutos \\
\hline Chopo & Cho’opó & Mimosa palmeri Rose & Tos, anginas y diarrea & Corteza \\
\hline Copal & Jecapí & Bursera stenophyllaSprague \& Riley & Tos, resfriado & Resina \\
\hline Copalquín & Jutetió & $\begin{array}{c}\text { Hintonia latiflora (Sesse \& Mocino ex } \\
\text { DC.) Bullock }\end{array}$ & $\begin{array}{l}\text { Falta de apetito, parásitos y } \\
\text { diabetes }\end{array}$ & Raíz \\
\hline Corcho & Juyépori & Diphysa suberosa S. Watson & Resfriado & Corteza \\
\hline Coronilla & & Berlandiera lyrata Benth. & Dolor de estómago & $\begin{array}{l}\text { Hojas y } \\
\text { ramas }\end{array}$ \\
\hline Damiana & & Turnera difusa Willd. ex Schult. & $\begin{array}{c}\text { Pasmo, problemas nerviosos, } \\
\text { afrodisiaco }\end{array}$ & Hojas \\
\hline Encino & Cusi & Quercus albocinata Trel. & Empacho y llagas & Corteza \\
\hline Etcho & Chiquí & $\begin{array}{l}\text { Pachuycereus pecten-aboriginum } \\
\text { Engelm. ex S. Watson Britton \& Rose }\end{array}$ & $\begin{array}{c}\text { Picaduras de alacrán, insectos y } \\
\text { sarna }\end{array}$ & Tallo \\
\hline Guacaporo & Guacaporo & Parkinsonia aculeata Linnaeus & Tos & Hojas \\
\hline Guamúchil & Maicochini & Pithecellobium dulce (Roxb.) Benth. & Dolor de estómago & Corteza \\
\hline Guásima & Agiyá & Guazuma ulmifolia Lam. & $\begin{array}{l}\text { Dolor de estómago, picadura de } \\
\text { alacrán }\end{array}$ & Corteza \\
\hline Guayacán & & Guaiacum coulteri A.Gray & Asma & Corteza \\
\hline Igualama & Juhuari & Vitex mollis Kunth & Problemas de parto & $\begin{array}{l}\text { Corteza, } \\
\text { raíz }\end{array}$ \\
\hline Mauto & Ma'a & $\begin{array}{l}\text { Lysiloma divaricatum (Jacq.) } \\
\text { J.F.Macbr. }\end{array}$ & Diarrea & Corteza \\
\hline Mezquite & Jupa'ara & Prosopis glandulosa Torr. & Mal de ojo, fiebre, sarampión & $\begin{array}{c}\text { Corteza, } \\
\text { hojas, } \\
\text { resina y raíz }\end{array}$ \\
\hline Mostaza & Guacharay & Dryopetalon runcinatum A. Gray & Dolor de espalda & Semillas \\
\hline Ocotillo & Chonolí & Fouquieria macdougallii Nash & Resfriado, susto & Hojas, rama \\
\hline Palo Brasil & Huichilaco & Haematoxylum brasiletto Karsten & $\begin{array}{l}\text { Mensutración, riñones y } \\
\text { curculación }\end{array}$ & Corteza \\
\hline Palo colorado & Hueraquí & Caesalpinia platyloba S. Watson & Llagas & Raíz \\
\hline
\end{tabular}


Continuación

\begin{tabular}{|c|c|c|c|c|}
\hline Nombre común & $\begin{array}{l}\text { Nombre } \\
\text { makurawe }\end{array}$ & Nombre científico & Enfermedad & Parte útil \\
\hline Palo mulato & Mulato & Bursera grandifolia (Schltdl.) Engl. & Cortaduras & Corteza \\
\hline Palo santo & Tochiguó & $\begin{array}{l}\text { Ipomoea arborescens (Humb. \& } \\
\text { Bonpl. ex Willd.) G. Don }\end{array}$ & $\begin{array}{c}\text { Picadura de alacrán, mordedura } \\
\text { de víbora }\end{array}$ & $\begin{array}{l}\text { Savia, } \\
\text { corteza }\end{array}$ \\
\hline Palo zorrillo & Juracosí & $\begin{array}{c}\text { Senna atomaria (L.) L.H.S. Irwin \& } \\
\text { Barneby }\end{array}$ & Dolor de cabeza & Hojas \\
\hline Papache borracho & Cabijósocura & Randia obcordata S. Watson & Parásitos & Fruto \\
\hline Papache & Josocura & Randia echinocarpa S. Watson & Parasitos, dolor de muela y llagas & Fruto, raíz \\
\hline Pionia & Mayomecha & Lashiathaea podocephala & Empacho & Raíz \\
\hline Pochote & Guacapí & Ceiba acuminata (S. Watson) Rose & Dolor de estómago & Corteza \\
\hline Rama de toro & Pipichiaba & Tetramarium sp. & Malaria y susto & Raíz \\
\hline Rama quemador & Taigual, tejéhuari & Tragia nepetifolia Cav. & Dolor de estómago & Hojas \\
\hline Salvia & Bibinó & Hyptis albida Kunth & $\begin{array}{c}\text { Parto, cólicos, dolor de cabeza, } \\
\text { relajante }\end{array}$ & $\begin{array}{l}\text { Hoja, raíz y } \\
\text { rama }\end{array}$ \\
\hline Sávila & & Aloe vera $\mathrm{L}$. & Infecciones & Pulpa \\
\hline Sibiri & Sevelí, sahueri & Opuntia thurberi Engelm & Dolor de estómago, diarrea & Fruto \\
\hline Tepeguaje & Machahuí & Lysiloma watsonii Rose & Gastritis, Ilagas & Corteza \\
\hline Toloache & Tecuyabí & Datura discolor Bernhardi & Infecciones, inflamaciones & Hojas \\
\hline Torote & To'oro & Bursera laxiflora S. Watson & Cortaduras & Corteza \\
\hline Vara blanca & Sejcó & Croton fantzianus F. Seymour & Empacho & Raíz \\
\hline Yerba del indio & & Aristolochia quercetorum Standley & Empacho & Raíz \\
\hline
\end{tabular}

Para los guarijío, el concepto de salud encierra un conjunto de significados que incluyen no sólo no estar enfermo, sino la capacidad de poder trabajar, de poder comer y de estar contento [14]. La salud es estar bueno y sano, es estar bien, con buena cara y buena fuerza, poder cumplir la obligación, puesto que el que está sano se siente bien, no está triste, está bien con su familia, con su comunidad y uno que está bueno y sano está alegre está bien con Dios y los demás. La salud y la enfermedad son conceptos que están estrechamente relacionados con la concepción que tienen respecto a su vida como individuo y como parte de una sociedad. Para los guarijío, el individuo es importante como miembro del todo. Consideran que su vida no está separada de la existencia de las plantas, de los ríos, de los animales y demás elementos naturales, sino que toda ella depende de la concordancia con cada uno de ellos. Es una concepción integral del mundo donde se entrelazan aspectos biológicos, sociales, religiosos y culturales.

En la medicina tradicional guarijío, los conceptos de balance y equilibrio constituyen nociones fundamentales que se interpretan en sentido físico, psíquico, mítico moral y religioso. La enfermedad va más allá de las causas meramente biológicas; aspectos como el coraje, la ansiedad, la tristeza, la envidia, el susto o el "mal puesto", son también causas de enfermedad. La falta de respeto a sus semejantes, a la naturaleza, a las tradiciones, costumbres y creencias religiosas también son causas de enfermedad. En la cuadro 2 se muestran las plantas útiles en la medicina tradicional. 
Cuadro 3. Las plantas en la construcción y vivienda de los Makurawe/Guarijío de Sonora.

\begin{tabular}{|c|c|c|c|}
\hline Nombre común & $\begin{array}{l}\text { Nombre } \\
\text { makurawe }\end{array}$ & Nombre científico & Usos \\
\hline Algodoncio & Machicari & Wimmeria mexicana (DC.) Lundell & Vigas y postes \\
\hline Amapa & Tabuió & Tabebuia impetiginosa (Mart. ex DC.) Standl. & Insecticida, vigas, horcones \\
\hline Batamote & Guachomó & Baccharis salicifolia (Ruiz \& Pavon) Persoon & Techos y trampas para pescar \\
\hline Cacachila & Jimuari, imora & $\begin{array}{l}\text { Karwinskia humboldtiana (Willd. ex Roemer \& } \\
\text { Schultes) Zucc. }\end{array}$ & Postes y horcones \\
\hline Chalate & Guagüurí & Ficus trigonata L. & Bateas y tazones \\
\hline Chopo & Cho'opó & Mimosa palmeri Rose & Postes \\
\hline Copal & Jecapí & Bursera stenophylla Sprague \& Riley & Comederos y canaletas (ganado) \\
\hline Copalquín & Jutetió & Hintonia latiflora (Sesse \& Mocino ex DC.) Bullock & Postes y vigas \\
\hline Encino & Cusi & Quercus albocinata Trel. & Postes y horcones \\
\hline Etcho & Chiquí & $\begin{array}{c}\text { Pachuycereus pecten-aboriginum Engelm. ex S. } \\
\text { Watson Britton \& Rose }\end{array}$ & Mesas, vigas, tarimas, harpas \\
\hline Guamúchil & Maicochini & Pithecellobium dulce (Roxb.) Benth. & Bancos y vigas \\
\hline Guásima & Agiyá & Guazuma ulmifolia Lam. & $\begin{array}{l}\text { Muebles, taburetes, instrumentos } \\
\text { musicales }\end{array}$ \\
\hline Mauto & Ma'a & Lysiloma divaricatum (Jacq.) J.F.Macbr. & Horcones y postes \\
\hline Mezquite & Jupa'ara & Prosopis glandulosa Torr. & Vigas y horcones \\
\hline Palma & Tajcú & Sabal uresana Trel. & $\begin{array}{l}\text { Petates, angarias, sombreros, } \\
\text { canastas, techo }\end{array}$ \\
\hline Palo Brasil & Huichilaco & Haematoxylum brasiletto Karsten & Postes y horcones \\
\hline Palo colorado & Hueraquí & Caesalpinia platyloba S. Watson & Vigas y postes \\
\hline Palo de asta & Camahuí & Cordia sonorae Rose & Mangos para herramientas de trabajo \\
\hline Palo fierro & Huorijtá & $\begin{array}{l}\text { Choloroleucon mangensex (Jacq.) Britton \& Rose, N. } \\
\text { Amer. }\end{array}$ & Postes, vigas y horcones \\
\hline Palo santo & Tochiguó & $\begin{array}{c}\text { Ipomoea arborescens (Humb. \& Bonpl. ex Willd.) G. } \\
\text { Don }\end{array}$ & Insecticida \\
\hline Palo zorrillo & Juracosí & Senna atomaria (L.) L.H.S. Irwin \& Barneby & Postes y horcones \\
\hline Pino & Guaj'co & Pinus oocarpa Schiede ex Schltdl. & Techo y vigas \\
\hline Pochote & Guacapí & Ceiba acuminata (S. Watson) Rose & Vigas, comederos, puertas \\
\hline Tchuna & Huilocochi & Ficus insipida Willd. & Platones y artesanías \\
\hline Tepeguaje & Machahuí & Lysiloma watsonii Rose & Bateas, postes, vigas \\
\hline Torote & To'oro & Bursera laxiflora S. Watson & Mascaras pascola, platos y tazones \\
\hline Vara blanca & Sejcó & Croton fantzianus F. Seymour & Escobas, vigas y techos \\
\hline
\end{tabular}


Un pedazo de sierra. La vivienda de los Guarijío. Sólo basta mirar una de sus viviendas para darse cuenta de su apego a la sierra y de la interacción constante con su medio natural. En cada uno de los rincones de sus casas, pareciera como si hubiesen trasladado un pedazo del monte. Las viviendas generalmente constan de un cuarto y una ramada. Estos espacios están construidos básicamente con piedras, barro y plantas. El techo es de terrado y el piso de tierra; en la ramada (área abierta con techado de ramas), podemos observar una serie de muebles y enseres que nos hablan de la importancia de las plantas en sus actividades domésticas: la hornilla, el molino de mano y la olla del agua que descansan sobre una horqueta hecha de guásima (Guasima ulmifolia). En la ramada se preparan los alimentos, se teje la palma o se afina el arpa y el violín. Una cruz hecha con hojas de álamo o sauce protege la vivienda guarijío de los "malos vientos".

En la vivienda las plantas adquieren distintas formas. Los guarijío, con gran creatividad, han transformado los troncos de las plantas en una multiplicidad de enseres domésticos y herramientas de trabajo: tarima, tapancos, mesas, sarso, tapésti, sillas, petates, canastas, angarías, puertas, cercos, y hasta escobas. Conocen la calidad de la madera y seleccionan la más adecuada para cada tipo de mueble o herramienta de trabajo. En el cuadro 3 se pueden observar las plantas útiles para la construcción.

Tramando la Palma. Las plantas en las artesanías. La palma (Sabal uresana) es la planta básica con la que los guarijío elaboran sus artesanías. La palma se corta casi todo el año, excepto en los tiempos de lluvia. Los hombres son los encargados de traer la palma y las mujeres se dedican a tejer. La parte de la planta con la que se tejen estas artesanías es el cogollo (hoja tierna). El oficio de artesana se aprende desde la niñez; las mujeres saben con exactitud la cantidad de hojas que se necesita para confeccionar las distintas artesanías.

Las plantas en las fiestas. Las fiestas son espacios esenciales en la vida de las comunidades de los Guarijío. Los motivos y elementos con que se llevan a cabo, son una clara evocación a la naturaleza y confirman de nuevo su apego a la sierra y a las distintas especies que viven en ella. La Tuburada y la Cava-Pisca son las celebraciones más importantes. La tuburada se lleva cabo a partir de una promesa, ofrecida para asegurar la salud, proteger la siembra o para pedir por la lluvia. Está directamente vinculada con compromisos existenciales para todos y cada uno de los Makurawe. La Cava-Pisca tiene un sentido más comunitario, se orienta al ciclo agrícola en función del cual muchos aspectos de la vida cotidiana y el sentido ritual son expresados, agradeciendo por un lado la cosecha del año anterior y pidiendo o invocando porque el próximo también sea benévolo. Las fiestas reflejan de nuevo la cosmovisión del mundo desde los ojos de los Guarijío [7].

Las fiestas poseen una centralidad en la cultura de los Guarijío; son la expresión fundamental de su concepción religiosa. En ellas se ve reflejado el culto a lo sagrado, a lo divino. Se hacen porque Dios así lo quiso, y se conservan como una forma de respeto y agradecimiento a él como creador de la naturaleza de las plantas y los animales. Existe la creencia de que si dejan de celebrarlas todo el mundo desaparecerá y lo llevan en el nombre: Makurawe que significa "Ios que amacizan o pisan la tierra" o también "Ios que agarraron las piedras". Las mujeres son las encargadas de amacizar la tierra.

De nuevo, en estos importantes eventos, las plantas están presentes y tienen un significado profundo en la cultura e identidad Guarijío: la guásima, (Guazuma ulmifolia) el etcho, (Pachycereus pecten-aboriginum) el bule (Langenaria sicerar) el copal, (Bursera lancifolia) el macucho (Nicotiana rustica) y el maíz son algunas de las especies más importantes en la fiesta de la tuburada. Los cantos son una clara evocación a la naturaleza: se le canta al palo verde, al lobo, a la avispa, al sauz y a la colmena. Los sones que tocan en las fiestas son al, mapache, maguey, carrizo, iguana, tecolote, coyote, zopilote, gavilán y ardilla. 
A continuación se muestran los resultados de los talleres comunitarios realizados en la población de Mesa Colorada. Los participantes enlistaron 63 plantas utilizadas en la comunidad; a partir de esta selección los guarijío hicieron un ejercicio de jerarquización que les permitió reflexionar en torno a la importancia de las plantas en su vida cotidiana. Cabe señalar que esta acción resultó en cierta forma complicada, pues para los Guarijío todas las plantas son importantes. Sin embargo, tras llegar a un consenso, pudieron jerarquizar y enlistar aquellas plantas que desde su percepción depende más su sobrevivencia biocultural.

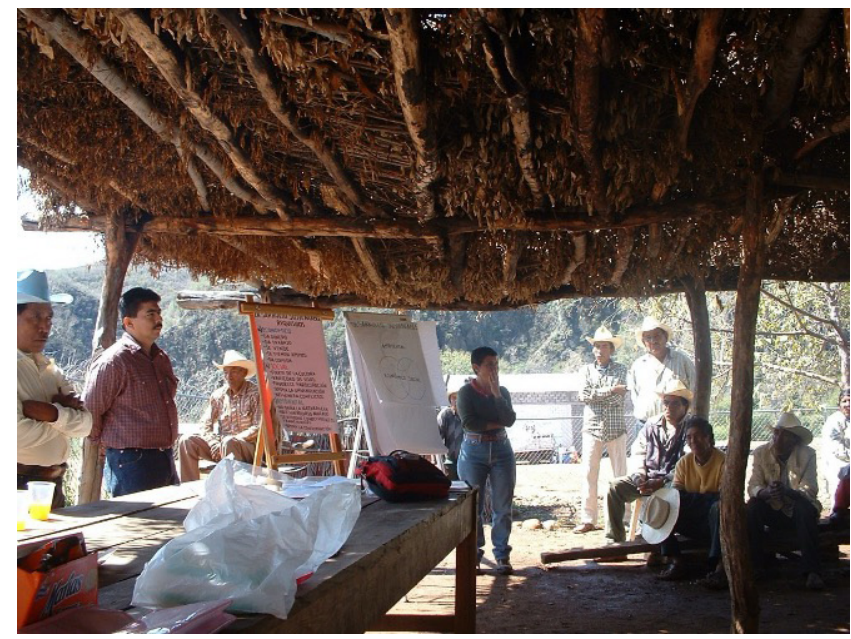

Figura 4. Taller de participación comunitaria.

Cuadro 4. Jerarquización de las plantas más utilizadas por los makurawe/guarijío.

\begin{tabular}{|c|c|c|c|}
\hline Nivel & Nombre común & N. Guarijío & Nombre científico \\
\hline 1 & Etcho & Chiquí & Pachuycereus pecten-aboriginum Engelm. ex S. Watson Britton \& Rose \\
\hline 2 & Guásima & Agiyá & Guazuma ulmifolia Lam. \\
\hline 3 & Jupa'ara & Mezquite & Prosopis glandulosa Torr. \\
\hline 4 & Palo Brasil & Huichilaco & Haematoxylum brasiletto Karsten \\
\hline 5 & Palma & Tajcú & Sabal uresana Trel. \\
\hline 6 & Vara blanca & Sejco & Croton fantzianus F. Seymour \\
\hline 7 & Amapa & Tabuió & Tabebuia impetiginosa (Mart. ex DC.) Standl. \\
\hline 8 & Guamúchil & Maicochini & Pithecellobium dulce (Roxb.) Benth. \\
\hline 9 & Pochote & Guacapí & Ceiba acuminata (S. Watson) Rose \\
\hline 10 & Chopo & Cho' opó & Mimosa palmeri Rose \\
\hline 12 & Amapa & Tabuió & Tabebuia impetiginosa (Mart. ex DC.) Standl. \\
\hline 13 & Palo Colorado & Hueraquí & Caesalpinia platyloba S. Watson \\
\hline 14 & Palo de Asta & Camahuí & Cordia sonorae Rose \\
\hline 15 & Chani & Comba'ari & Hyptis suaveolens (L.)Poit. \\
\hline 16 & Papaches & Josocura & Randia obcordata y R. Echinocarpa S. Watson \\
\hline 17 & Chiltepín & Co’co’rí & Capsicum annum var. glabriusculum (Heiser y Pickersgill) \\
\hline 18 & Saya & & Amoreuxia cf. palmatifida Sesse \& Mocino ex DC. \\
\hline 19 & Salvia & Bibinó & Hyptis albida Kunth \\
\hline 20 & Chichiquelite & Maneyoqui & Solanum americanum Miller \\
\hline 21 & Quelite & Huehué & Amaranthus leucocarpus S. Watson \\
\hline
\end{tabular}




\section{Reflexiones finales}

A través de este trabajo se ha intentado resaltar la importancia de las plantas entre las formas de subsistencia del pueblo Makurawe/Guarijío de Sonora. Los resultados revelan que las plantas han sido esenciales para su sobrevivencia biológica y cultural. Este pueblo ha sabido convivir con la sierra de una manera armónica. En esta interacción, se observa una visión holística: las plantas no son imaginadas como individuos biológicos o materias primas aisladas sino más bien están consideradas dentro de una matriz integrada que engloba aspectos ecológicos, económicos y socioculturales tales como: costumbres, creencias, religión, festividades, cantos, mitos, leyendas y cosmogonía. De modo tal, que las plantas en el pensamiento de los guarijío, están enredadas e integradas en las esferas del desarrollo sostenible: el ámbito biológico, social, cultural y económico.

Es necesario revalorar y recuperar el conocimiento tradicional de nuestros pueblos indígenas, no sólo con el propósito de retomarlo para fines utilitarios, sino por el papel que juega en la realidad socio ambiental. Los saberes de los grupos indígenas con respecto a los recursos naturales podrían contribuir de manera importante en el establecimiento de una propuesta alternativa ante la crisis social y ambiental que hoy enfrentamos. Esto es esencial si se consideran, entre otras, las modificaciones en sus formas de alimentación que están afectando su salud y el avance de proyectos modernizadores que amenazan su entorno natural y su propia permanencia biocultural.

Para lograrlo es necesario hacer un planteamiento enraizado en los saberes, la identidad y la cultura de los guarijío. De ahí debe surgir la savia que permita a los grupos indígenas tener un proyecto que los convierta en participantes activos sobre sobre las decisiones que atañen a su propio destino. En el desarrollo de este trabajo, los guarijío identificaron algunas plantas que desde su cosmovisión podrían ser utilizadas como bases de proyectos alternativos de desarrollo sustentable. Esta propuesta requiere de seguir avanzando en la construcción de espacios para el diálogo horizontal de saberes donde se articulen los grupos indígenas, la academia y los gobiernos. La etnobotánica pudiera convertirse en una disciplina que logre tender puentes entre los eslabones del saber tradicional y el conocimiento académico, que al final repercuta en el bienestar de estos pueblos. De no hacerlo se seguirán perdiendo infinidad de recursos bioculturales desconocidos por la ciencia.

\section{Agradecimientos}

A esos hombres y mujeres de rostros con destellos rojizos apenas visibles para muchos sonorenses. A los que luchan pacientes y tenaces por sus diferencias, por su sierra, por justicia social. A los que alimentan la Tierra con sus saberes, danzas, cantos y rezos. A los que purifican la tierra con copal para que dure más, para que dé más frutos, para que baje el agua. A los Guarijío-Makurawe de Sonora. Finalmente, a la Arquitecta Daniela Castillo Bañuelos por el apoyo en la edición gráfica

\section{Referencias}

[1] E. Boege, El patrimonio biocultural de los pueblos indígenas de México. Hacia la conservación in situ de la biodiversidad y agrodiversidad en los territorios indígenas, Instituto Nacional de Antropología e Historia. Comisión Nacional para el Desarrollo de los Pueblos Indígenas, México, 2008.

[2] N. Touche, "La madre tierra pierde sus árboles de manera acelerada", El economista, México, 23-abril del 2015 (En línea) Disponible: https://www.eleconomista.com.mx/arteseideas/La-madre-tierra-pierde-susarboles-de-manera-acelerada--20150421-0177.html 
[3] S. Del Amo, "El cambio de Modelo de Desarrollo Necesario en un país Pluricultural. Algunos Aspectos que se deben tomar en cuenta", Etnobiología Volumen (9) No.1 pp.60-76, 2011.

[4] J. Vélez y C. Harris, Pueblos Indígenas del México Contemporáneo. Guarijíos. 2004. (En línea), Disponible: http://www.cdi.gob.mx.

[5] INEGI, Instituto Nacional de Geografía y Estadística. Censo de Población y Vivienda. Aguascalientes, México. 2010.

[6] J.L Moctezuma y A. Aguilar, Los Pueblos Indígenas del NOROESTE. Atlas Etnográfico. Ciudad de México. Instituto Sonorense de Cultura/ Instituto Nacional de Lenguas Indígenas/ Instituto Nacional de Antropología, México, 2013.

[7] A. Aguilar, Los Ritos de la identidad: ritualidad, diversidad y estrategias de resistencia indígena en el noroeste de México. (Tesis de maestria. Escuela Nacional de Antropología), Ciudad de México, 1998.

[8] A. Aguilar, "Macurawe, los guarijío". Revista de Estudios sociales de Investigación del Nororeste, Volumen VI. No. 12., pp. 144-162, México, 1996.

[9] D. Yetman, The Guarijios of the Sierra Madre. Hidden People of Northwestern Mexico, Alburquerque. USA. Unversity of Arizona Southwest-University of New Mexico, 2002.

[10] A. Martínez, R. Felger y A. Búrquez, Los ecosistemas terrestres: Un diverso Capital Natural. En F. Molina y T. Vandevender T. (Editores). DIVERDSIDAD BIOLÓGICA EN SONORA. Ciudad de México. Universidad Nacional Autónoma de México. Comisión Nacional para el Conocimiento y Uso de la biodiversidad, 2010. pp. 129-137.

[11] V. Salazar y P. L. Salido, El contexto regional guarijío. Un encuentro con la pobreza. Revista de Estudios sociales de Investigación del Nororeste. Volumen VI. (No. 12), México, 1996, pp. 39-66.

[12] H. S. Gentry, "The Warihío Indians of Sonora-Chihuahua: An Etnographic Survey", Anthropological Papers No. 65. Bulletin 186. Washington. Bureau of American Ethnology, 1963.

[13] M. Gómez, "Saber Indígena y Medio Ambiente: Experiencias de Aprendizaje Comunitario". En Leff, E. (Coordinador). La Complejidad Ambiental. Ciudad de México. Siglo XXI. 2003.

[14] J. A. Haro, B. Lara, M. R. Palacios, V. Salazar V. y P. L. Salido, El sistema local de Salud Guarijío-Makurawe. Un modelo para Construir. Hermosillo. El Colegio de Sonora, CIAD, CONACULTA, UNISON, INI. pp. 273-209, 1998. 